\title{
Urologic management of the transgender patient
}

With growing social acceptance and increasing access, transgender care is at the forefront of medicine. Urologists, especially those of us dedicated to genitourinary reconstruction, stand to play a major role in the delivery of surgical care. The challenges of primary gender-affirming surgery include the lack of formal urologic training, the historically siloed method of delivery of care, and the relative lack of comparative research regarding optimal techniques. Understanding gender-affirming surgery (genital and non-genital) is critical even to those reconstructive urologists who do not perform primary gender-affirming surgery because they may be faced with addressing the complications of primary surgery. In performing revision surgery, one needs a broad understanding of the multiple techniques for primary gender-affirming surgery to identify pitfalls and potential complications. Regardless, complication rates are high, and revisions may not be successful. We seek to provide a review of the urologic management of the transgender patient, drawing from an international set of experts with varying experiences.

We begin with a broad review of epidemiology, trends and surgical principles in the gender affirming surgery. In an effort to be comprehensive, non-urologic procedures, such as chest and craniofacial surgery, are presented along with fertility concerns. Individual surgical procedures of vaginoplasty, metoidioplasty, and phalloplasty are then discussed. Finally, urologic consequences of phalloplasty, such as urethroplasty and penile prosthesis placement, are reviewed.

Respectfully, we offer some words of caution. It is important for those surgeons who wish to get involved in transgender care to become trans-competent and immerse themselves in the field. The procedures are technically demanding, and patients' individual desires and anatomy add constant challenges to a set of procedures with high complication rates. Even in the portions of each surgery that overlap with existing urologic procedures (e.g., orchiectomy and penectomy during vaginoplasty), there are nuances specific to the transgender patient, which are realized with training and experience. Thus, we would warn readers to avoid "dabbling" in gender-affirming surgery. Additionally, the surgical care of transgender patients is multidisciplinary. It is important for the urologist to work within a team of other specialties (plastics, colorectal, etc.) capable of handling the various surgical complications and the non-surgical workload (dilation, letters of support, insurance issues, etc.) that come with delivery of care to the transgender patient. Regardless, our goal was to provide a timely and salient set of articles for urologists either delving into transgender care or those well-entrenched in the field.

\section{Acknowledgments}

None.

\section{Footnote}

Conflicts of Interest: SP Elliott: Consultant to Boston Scientific. Investment interest in Percuvision. Paid investigator for Urotronic. The other authors have no conflicts of interest to declare.

Ethical Statement: The authors are accountable for all aspects of the work in ensuring that questions related to the accuracy or integrity of any part of the work are appropriately investigated and resolved. 


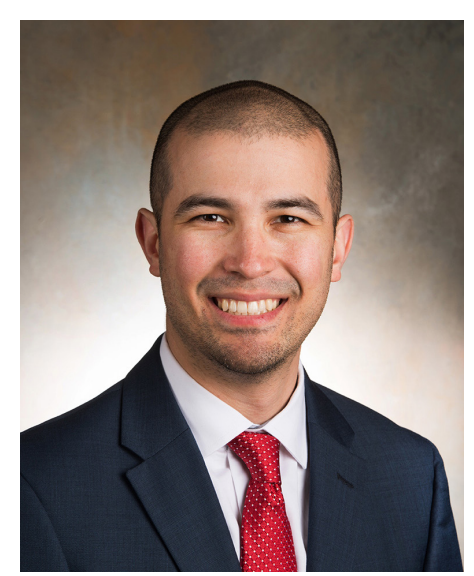

Joseph J. Pariser

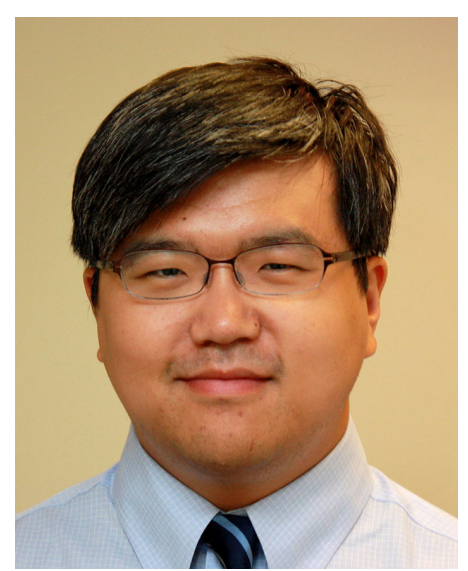

Lee C. Zhao

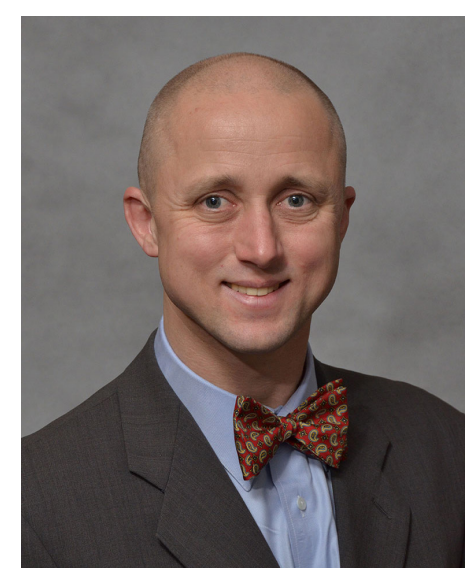

Sean P. Elliott

Joseph J. Pariser, MD

Department of Urology, University of Minnesota, Minneapolis, MN, USA.

(Email:jpariser@umn.edu)

Lee C. Zhao, MD

Department of Urology, New York University Langone Health, New York, NY, USA.

(Email:Lee.Zhao@nyumc.org)

Sean P. Elliott, MD

Department of Urology, University of Minnesota, Minneapolis, MN, USA.

(Email: selliott@umn.edu)

doi: $10.21037 /$ tau.2019.06.16

View this article at: http://dx.doi.org/10.21037/tau.2019.06.16

Cite this article as: Pariser JJ, Zhao LC, Elliott SP. Urologic management of the transgender patient. Transl Androl Urol 2019;8(3):182-183. doi: 10.21037/tau.2019.06.16 